\title{
INNOVACIÓN SOCIAL, EXPERIENCIA DE VINCULACIÓN CON LA SOCIEDAD. SANTA ANA. MANABÍ. ECUADOR
}

\author{
AUTORES: Claribel Silvia González Calzadilla ${ }^{1}$ \\ Manuel de Jesús Andérez Velázquez ${ }^{2}$ \\ Karen Lorena Briones Cedeño ${ }^{3}$
}

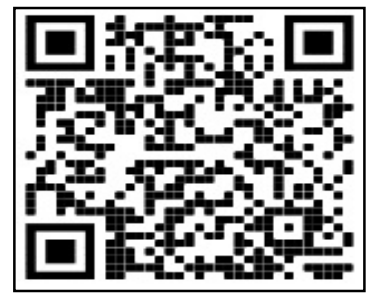

DIRECCIÓN PARA CORRESPONDENCIA: claribel.gonzalez@utm.edu.ec

Fecha de recepción: 20/08/2020

Fecha de aceptación: 22/12/2020

\section{RESUMEN}

El trabajo muestra la experiencia realizada en el Cantón Santa Ana .Manabí donde se aprecia la vinculación con la sociedad del centro coordinador de las investigaciones agropecuarias (CIAM) en dos Universidades, la Técnica de Manabí (UTM) del Sur de Manabí (UNESUM) con el centro de desarrollo local CECADEL (hoy Campus de Santa Ana), en la importancia de una innovación social puesta en práctica de forma novedosa, creativa y transformadora aplicada en sectores vulnerable como son: los adolescentes y jóvenes, los adultos mayores y los discapacitados. La metodología utilizada fue el método de análisis y síntesis de diferentes documentos legales que permitieron un trabajo coherente, también el análisis cualitativo, en la aplicación de la técnica, acción- participación, con diversas acciones que se desarrollaron con el objeto de estudio, las actividades dirigidas a la capacitación, el fomento y preparación de líderes comunitarios, se exponen en el trabajo. Los resultados evidencian que el esquema adoptado y las formas organizativas propuestas en su aplicación detectan un conjunto de problemas, a los cuales se les elabora la propuesta de proyectos que fortalecieron la vinculación de la Universidad con la alcaldía, su entorno parroquial y comunitario, favoreciendo la capacitación y la preparación de sectores objeto de estudio en temas de cultura general e integral. Se concluye con las propuestas organizativas a través de proyectos los que constituyen entornos de un trabajo de innovación social

PALABRAS CLAVE: innovación social comunitaria, Vinculación -Universidad

\footnotetext{
${ }^{1}$ Lic.Mg.C.Docente.Universidad Técnica de Manabí. Santa Ana. Manabí. Ecuador.orcid.0000-0001-8744-0874 Email: claribel.gonzalez@utm.edu.ec

2 Ing. Dr. Manuel de Jesús Andérez Velázquez investigador auxiliar, profesor auxiliar. Universidad Técnica de del Sur de Manabí. Ciudad Santa Ana, Manabí, Ecuador .manuel.andérez@unesum.edu.ec

3Lic.Karen Lorena Briones Cedeño Universidad Técnica de del Sur de Manabí. Ciudad Santa Ana, Manabí, Ecuador.keren.lorena@unesum.edu.ec
}

(C) Universidad Estatal del Sur de Manabí. Jipijapa, Ecuador. 
Resabala Claribel Silvia González Calzadilla; Manuel de Jesús Andèrez Velázquez; Karen Lorena Briones Cedeño

\title{
SOCIAL INNOVATION, EXPERIENCE OF BONDING WITH SOCIETY IN SANTA ANA. MANABÍ, ECUADOR
}

\begin{abstract}
The work shows the experience carried out in the Canton Santa Ana. Manabí where the link with the society of the Coordinating Center of Agricultural Research (CIAM (in two Universities, the Technique of Manabí (UTM) of the South of Manabí (UNESUM) with the local development center CECADEL (today Santa Ana Campus), on the importance of a social innovation put into practice in a novel, creative and transformative way applied in vulnerable sectors such as: adolescents and young people, the elderly and the disabled. The methodology used was the method of analysis and synthesis of different legal documents that allowed a coherent work, also qualitative analysis, in the application of the technique, action-participation, with various actions that were developed with the object of study, the activities aimed at training, fostering and preparing community leaders, are exposed in the work. The results show that The scheme adopted and the organizational forms proposed in its application detect a set of problems, for which the proposal of projects that strengthened the University's link with the mayor's office, its parish and community environment, favoring training and education, is elaborated. preparation of sectors under study on issues of general and comprehensive culture. It concludes with the organizational proposals through projects which constitute environments of a social innovation work
\end{abstract}

KEYWORDS: Community social innovation, Bonding -University

\section{INTRODUCCIÓN}

Una definición mínima de innovación social, que goza de consenso, es la de Moularet (2009) para quien se trata de un proceso orientado a la satisfacción de las necesidades humanas cuando supone, además del cambio sustantivo, una transformación de las relaciones sociales que mejoren los sistemas de gobernanza y que establezcan nuevas estructuras y organizaciones. Es decir, que resuelve la satisfacción de las necesidades humanas básicas e innova en las relaciones sociales entre personas y grupos en las comunidades de afectados. Herrera (2016.)

Esta definición nos confirma que el trabajo de organización con grupos vulnerables, estableciendo con ellos un intercambio, que los haga reflexionar a la vez que los capacite en temas de conocimiento de su entrono de forma multidisciplinaria, produce cambios en las personas donde las mismas participan, brindando soluciones a su entorno, es a lo que a criterio de los autores constituye una innovación social.

Y se coincide con que la universidad debe constituirse en la aliada celosa del devenir histórico, actual y perspectivo de la emergencia ciencia-tecnología -sociedad, a través de un pensamiento reflexivo-comprensivo que permita interpretar adecuadamente la incidencia y efecto del conocimiento, ciencia y la tecnología en la sociedad actual (Figaredo, 2009). 
Por ellos, las formas organizativas adoptadas en la experiencia que se muestra permiten a través de la selección de la misma en orden de aparición la comparación entre la Forma organizativa/vinculación y los resultados, en cinco pasos específicos.

Por otra parte, sirvió de mucho tener en cuenta en la experiencia señalada por De la Torre (2013) cuando dice que: es importante referir que las funciones sustantivas de la universidad como trasmisora del conocimiento, fundamentalmente a través de la docencia como refiere, la investigación, así como también la generación y aplicación del nuevo conocimiento con la investigación; también, finalmente a la difusión y extensión del conocimiento, con la vinculación universidad-sociedad. Es decir que el apoyo que brinda la universidad por todo lo que representa para la comunidad proporciona una parte importante en lograr innovación social en la comunidad.

La educación superior ecuatoriana ha sugerido y orientado a todas las IES del país dedicarle mayor atención a la vinculación con la sociedad, su transformación y llevarla a la sostenibilidad (LOES 2018)

Por lo tanto, la educación superior ecuatoriana no ha estado exenta de los cambios ocurridos en la educación superior y ha trabajado en el desarrollo de estos cambios. Por lo antes citado, constituye una temática de actualidad en las IES ecuatorianas trabajar sistemáticamente con las comunidades de su entorno en coordinación con los GAD cantonales y parroquiales, así como con las demás estructuras del Estado y Sociedad conllevando esto al desarrollo local innovador.

La vinculación con la sociedad, junto con la docencia y la investigación, constituye además de la gestión en los pilares de la academia Andèrez y col. 2020, por una parte, la docencia es fundamental pues establece la pertinencia de las universidades como correspondencia entre las necesidades de la sociedad y los perfiles académicos de la formación de los profesionales, por otra, la investigación muestra cómo se corresponde la enseñanza con las problemáticas que tiene y necesita la sociedad que se investigue, resuelva y socialice. Aquí debe estar presente la participación .de los estudiantes, los docentes investigadores y la sociedad que además de participar debe recibir estos resultados y de conjunto socializarlos, innovar y potenciar sus alcances. Todo lo anterior estaría dado a la espontaneidad si la gestión universitaria no apoya y acompaña el proceso.

Las relaciones U-S(universidad-Sociedad) se suceden de formas diferentes:

-En algunas ocasiones se desarrollan mediante proyectos curriculares, en otras no necesariamente o por lo menos se relacionan directamente con este proceso.

-Existen otras formas en que la vinculación surge dentro de los programas académicos y comprometen un conjunto de proyectos curriculares

-Por otra parte, lograr una relación desde la academia fortalece también esta pertinencia social, cuando la Universidad se vincula con sectores sociales vulnerables, impulsa la extensión solidaria e investiga y trabaja para elevar la calidad de vida del conjunto de la población.

(C) Universidad Estatal del Sur de Manabí. Jipijapa, Ecuador. 
La experiencia que se expone se desarrolló en el cantón Santa Ana, ubicado geográficamente en el centro este de la provincia de Manabí, posee 2 parroquias urbanas y 4 rurales y 172 comunidades. La población aproximadamente según datos estadísticos del censo 2010 es de 47,385 hab, la rama de mayor concentración de la población activa está en la agricultura (50,16\%), por vocación tradicional, siguiéndole el comercio (8,74\%).

El trabajo se refiere a la pertinencia social, experiencia y los avances alcanzados desde las relaciones universidad - sociedad a los cual se le llama innovación social. Abordando en la primera parte el concepto de Centro de Capacitación y Desarrollo Local (CEDADEL) y un conjunto de ideas que la UNESUM ha venido desarrollando en los últimos dos años, enfatizando en su pertinencia con la sociedad, en la segunda parte se exponen las experiencias y características del mismo en el Cantón Santa Ana donde en dos etapas se han desarrollados los diferentes cursos de capacitación que han derivado a propuestas de proyectos que tributan al desarrollo de su Comunidad, Parroquia y Cantón .Las capacitaciones realizadas fortalecen la necesidad de propiciar la perspectiva social que tiene la academia y el propósito de beneficiar y fortalecer la vinculación de la Universidad con la alcaldía, su entorno parroquial y comunitario, favoreciendo el desarrollo local.

La temática de vinculación a la sociedad hoy en la actualidad en el campo universitario posee mucha connotación, se hace referencia a que esta vinculación se acerque cada día más al desarrollo local, en la mirada del grupo de trabajo se ha llevado este tema a la gestión del conocimiento función que juega la universidad en el desarrollo local y en este sentido el trabajo se identifica con lo señalado por Núñez (2016), donde apunta” una epistemología diferente :subraya que la producción, distribución y uso de los conocimientos y tecnologías tienen lugar siempre en contexto particulares, con sus singularidades económicas, culturales, valorativas y que ellos deben modelar sus prioridades y desarrollo.”(p.195)

El mundo en que vivimos parece depender cada vez más del conocimiento científico y tecnológico. Sin embargo, la forma en que nos relacionamos con este conocimiento depende de que la educación juegue un papel cada vez más relevante encaminado al entendimiento de la ciencia, la tecnología y la sociedad.

No siempre existe una comprensión muy clara de lo que es innovación social y generalmente cuando se hablar de innovación se refiere por lo general a la tecnológica, pero el concepto de innovar no es sólo hacer algo nuevo, es lograr materializarlo y comercializarlo exitosamente, lo que involucra no sólo la innovación tecnológica, sino también cambios organizacionales, gerenciales y comerciales y es ahí donde se encuentra específicamente la parte social.

Las experiencias que surgen en la comunidad constituyen un fenómeno de relevancia, así pues, en este marco teórico se analizan aportes de algunos autores que se entienden como innovación social, algunas de ellas aportan, al presente trabajo.

El concepto «innovación» viene resonando desde que Schumpeter (1961) lo acuñara para destacar la novedad técnica u organizacional en el proceso de producción, existiendo evidencias de su éxito en el ámbito empresarial y comercial. Recientemente cobra relevancia el concepto «innovación social» para explicar y buscar aquellas novedades que surgen a partir de la detección

168 UNESUM-Ciencias. Publicación cuatrimestral. Vol. 5, No. 1 (Enero-Abril), Año 2021 
de necesidades sociales. Sin embargo, se debe destacar su amplitud conceptual. María Rosa Herrera Gutiérrez (2016)

Ahora bien, para que existan cambios en el entorno comunitario con la innovación social deben verse transformaciones y estas pueden estar en correspondencia con las soluciones que se ofrezcan en este sentido se señala que: para que una experiencia de transformación tenga el impacto deseado es necesario crear conexiones profundas que ayuden a comprender la realidad de las personas, sea en el mundo rural o en un entorno urbano. transperiencias.com (2017)

Se determinaron como forma organizativa los diferentes pasos para lograr un cambio innovador por la experiencia: organización del trabajo con el GAD (Gobierno autónomo descentralizado), organización y selección de los grupos vulnerables con los que se trabajaría, planificación y organización de la capacitación, programa de los cursos y sus horarios, estructura organizativa sobre evaluación final y entrega de certificados, estas fueron las pautas que sentaron las bases desde el inicio en un diálogo abierto entre las universidades, las alcandía y algunas parroquias

\section{DESARROLLO}

Materiales y métodos

Una vez expuesto lo que se entiende por innovación social comunitaria, se analiza las formas organizativas propuestas que permiten ver la experiencia., basados en la preparación de los documentos normativos que existen sobre la vinculación de la universidad con la sociedad, el objeto de la investigación es dirigido a los participantes del curso de capacitación y el lugar de desarrollo o escenario es en el cantón Santa Ana

Primeramente, en el trabajo se utilizó el método de análisis y síntesis de diferentes documentos como: Ley Orgánica de Educación Superior (LOES), Arts. 8, 13, 88, 107; Plan Nacional de Vinculación de la Educación Superior con la Sociedad; y, Reglamento del Régimen Académico del CES, Arts. 6 y 77, y el Plan de Ordenamiento Territorial de Santa Ana. Asimismo, se trabajó con el Convenio marco de cooperación interinstitucional entre la Universidad Estatal del Sur de Manabí y la Universidad Técnica de Manabí 26/8/2015.

También, se tuvo en cuenta el análisis cualitativo, en la aplicación de la técnica, acción y participación, con diversas actividades que se desarrollaron en el objeto de estudio, en los intercambios en las diferentes capacitaciones que se realizaron, el listados de los estudiantes y finalmente las presentaciones de los proyectos que realizaron, las intencionalidades o propósitos así como el accionar de los involucrados en el trabajo permitieron el diagnóstico inicial a través del intercambio con los participantes del Cantón y algunas de sus parroquias, sobre los ejercicios que se realizarían en función de la capacitación desarrollada en el fomento y preparación de líderes comunitarios

Resultados y discusión

(C) Universidad Estatal del Sur de Manabí. Jipijapa, Ecuador. 
Resabala Claribel Silvia González Calzadilla; Manuel de Jesús Andèrez Velázquez; Karen Lorena Briones Cedeño

Organización de la vinculación con la sociedad

El Centro de Capacitación y Desarrollo Local (CEDADEL) de Santa Ana está muy ligado al Centro de Investigación Agropecuario de Manabí (CIAM), en el objetivo de ambas se encuentra la misión de Vinculación con la sociedad, en cómo trabajar para lograr cambios sustanciales ya sea en el conocimiento de los problemas de la realidad, como en el enfrentamiento de la población en temas de liderazgos visto en la perspectiva de contar con el relevo de la sociedad con mejor conocimiento, no solo de su entorno sino de la provincia y del país y para ello, se trabajó con el siguiente esquema reflejado en la Figura 1:

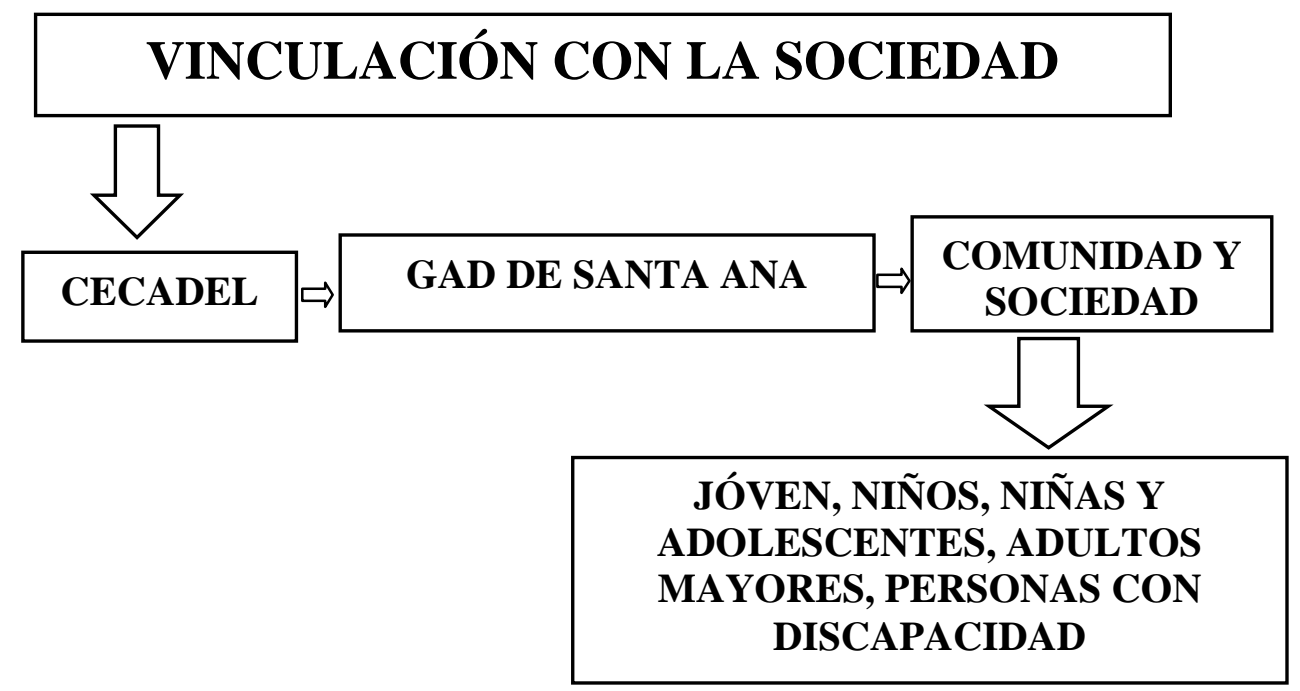

Figura 1: Gráfico que demuestra la vinculación del CECADEL-CIAM con la sociedad. Elaboración de los autores

Para la investigación como experimento se tomaron diferentes formas organizativas en las cuales se vería la vinculación que se realizaría con la sociedad (vista esta como la población del Cantón o las parroquias) y los resultados que se obtenían en cada una de las adoptadas. (Tabla1)

Tabla 1. Formas organizativas empleadas en el trabajo

\begin{tabular}{|l|l|l|}
\hline Forma & Vinculación & Resultado \\
\hline $\begin{array}{l}\text { 1.Organización del trabajo con } \\
\text { el GAD }\end{array}$ & El CECADEL-CIAM-GAD & $\begin{array}{l}\text {-Acercamiento de la } \\
\text { institución al GAD y sus } \\
\text { reuniones }\end{array}$ \\
\hline $\begin{array}{l}\text { 2.Organización con los grupos } \\
\text { vulnerables seleccionados }\end{array}$ & $\begin{array}{l}\text { El consejo cantonal de } \\
\text { protección de derechos .de } \\
\text { Santa Ana, CECADEL-CIAM }\end{array}$ & $\begin{array}{l}\text {-Definición de grupos de } \\
\text { trabajo } \\
\text {-Relación de coordinadores y } \\
\text { grupos vulnerables }\end{array}$ \\
\hline
\end{tabular}




\begin{tabular}{|l|l|l|}
\hline $\begin{array}{l}\text { 3.Planificación, organización } \\
\text { y capacitación }\end{array}$ & CECADEL, CIAM & $\begin{array}{l}\text {-Propuestas de cursos } \\
\text {-Se definen las horas clases y } \\
\text { horas que se ejecutarán }\end{array}$ \\
\hline $\begin{array}{l}\text { 4.Programa de los cursos y sus } \\
\text { horarios. Anexo1 }\end{array}$ & $\begin{array}{l}\text { CECADEL-CIAM con } \\
\text { dirección UNESUM }\end{array}$ & $\begin{array}{l}\text { Se definen temas específicos } \\
\text { para cada grupo vulnerable }\end{array}$ \\
\hline $\begin{array}{l}\text { 5. Estructura organizativa, } \\
\text { evaluación final y entrega de } \\
\text { certificado }\end{array}$ & $\begin{array}{l}\text { GAD-CECADEL-CIAM con } \\
\text { la Universidades UNESUM- } \\
\text { UTM y ULEAM }\end{array}$ & $\begin{array}{l}\text {-Elaborados proyectos que } \\
\text { responden a problemas de la } \\
\text { comunidad } \\
\text { Acto de graduación en Santa } \\
\text { Ana }\end{array}$ \\
\hline
\end{tabular}

Los objetivos que se perseguían con los cursos fueron:

1. Fortalecer la participación de la comunidad en la vida práctica de su cantón, parroquia y comunidad

2. Dotarlos de una preparación básica que le permita mirar con interés y conocimiento el entorno en su comunidad

3. Fortalecer valores en los participantes capaces de interactuar con sus realidades y actuar en consecuencia con sus valoraciones

4. Fomentar el interés investigativo.

5. Dotarlos de herramientas para la elaboración de proyectos de emprendimientos (de poca complejidad) con aportes al desarrollo local en sus parroquias

En una primera etapa: se efectuó sólo un curso de líderes para jóvenes, donde participaron 21 jóvenes. Presentando como evaluación final 8 proyectos que respondían, 4 al área de alimentos, 2 al medio ambiente y 2 sociales, simbolizaron una estrella que constituían especialidades de más prioridad según los temas de proyectos del primer módulo, (Estrella roja la alimentación, medioambiente azul y social los amarilla)

En la segunda etapa: los cursos ofertados, fueron 4 con 35 conferencias con temáticas de información general, destacando la preparación y la importancia que tiene en la formación de líderes comunitarios, visto estos como las personas que puedan asumir responsabilidades que actúan como guía o jefe de un grupo con capacidades de influir en otros sujetos, asumir tareas entre otras actividades, capaces de asimilar y ejecutar proyectos para contribuir con ellos al desarrollo local de sus comunidades.

Asistieron en total 64 personas desglosadas en: Jóvenes 11, adolescentes, niñas y niños 23, Adultos mayores 14 y personas con discapacidad 16 la forma de participación en los diferentes grupos fue distinta, presentaron proyectos sólo el grupo de Jóvenes del segundo módulo y las niñas, niños y adolescentes, en total 8 proyectos. 
Proyectos que se defendieron:

1. Uso diversificado de las frutas

2. Propuesta de eliminación en la quema indiscriminada en la parroquia Lodana

3. Utilización de la càscara de la naranja en velas

4. Modalidades de platos típicos ecuatorianos

5. Obtención de vinos de la grosella

6. Propuesta de la terminal terrestre de Santa Ana

7. Propuesta de conservación de la presa Posa Honda en la parroquia Honorato Vásquez

8. Trabajo comunitario dirigido al medioambiente en la parroquia Lodana

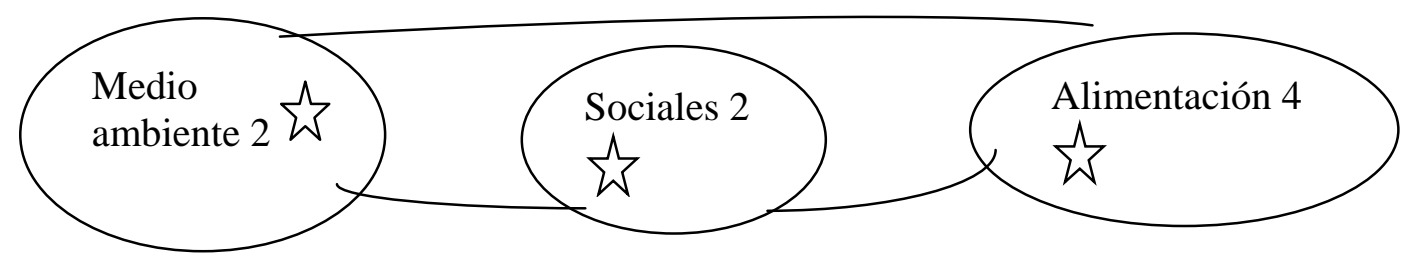

Figura 2. Representación por especialidades de la cantidad de proyectos presentados

Los cursos demostraron en la población vulnerable:

- Selección de temas de mucho interés que contribuyeron a enriquecer la cultura general integral

- Motivación en la comunidad por resolver los problemas de su entorno

- Apoyo del GAD cantonal y de los consejos parroquiales constituyó un eslabón determinante para el éxito de este trabajo

- Desarrollo de una sólida logística durante todo el programa para su éxito

- Existencia de un enorme potencial en la comunidad que se subestima con frecuencia, el cual se debe potenciar y multiplicar

- Las personas de la tercera edad o adultos mayores reúnen una experiencia ética, moral que no debe ser olvidada ni subvalorada. Es necesario aprovecharla y surgirán mejores ciudadanos comunitarios.

- Niños, niñas y adolescentes mostraron su sabiduría original, su entusiasmo, sus ansias de aprender, su constante curiosidad y franqueza de sus preocupaciones

- Ser capaces al final, de sugerir el diseñó de su tercer módulo para el siguiente. Con contenidos muy interesantes los cuales requieren ser preparados con todas las bases metodológicas, pedagógicas y didácticas.

172 UNESUM-Ciencias. Publicación cuatrimestral. Vol. 5, No. 1 (Enero-Abril), Año 2021 
Fue significativo que alumnos escogieran de las problemáticas a desarrollar los aspectos que afectan sus comunidades, los diseñaran y desarrollaran en su defensa final, como proyectos de investigación, lo que ratificó un sentido de pertenencia hacía su comunidad como entorno prioritario.

\section{CONCLUSIONES}

Se demuestra como, por un lado, se desarrolló la vinculación con la sociedad y por el otro, las formas organizativas desarrolladas como innovación social.

Cuando la Universidad se vincula con sectores sociales vulnerables, impulsa la extensión solidaria y contribuye a elevar la calidad de vida de la población como se establece con relación a los jóvenes, los adultos mayores, las personas con discapacidad, así como los niños, las niñas y los adolescentes alcanzándose una elevada pertinencia de la academia en el proceso de transformación del entorno.

Las formas organizativas adoptadas contribuyeron al incremento de los vínculos de la universidad con su entorno, lo que posibilitó la integración de la academia con el territorio o lo que es lo mismo Universidad-Sociedad

Los cursos impartidos como forma de vinculación con la sociedad se articularon coherentemente con el trabajo socio-profesional-universitario en la formación de líderes en los diferentes grupos vulnerables, los que permitirá hacerlos extensivos a otros cursos.

La aplicación de esta experiencia y el seguimiento de los procesos del trabajo investigativo demostró que se pueden aplicar diferentes formas organizativas de pertinencia social en la vinculación Universidad-Sociedad, avalado con los resultados alcanzados en la presentación de proyectos, para atenuar diferentes problemas y de esta forma se fortalece el desarrollo local

\section{REFERENCIAS BIBLIOGRÁFICAS}

Andèrez Velázquez, Manuel de J., González calzadilla, Claribel, Briones Cedeño, Karen 2020 La formación de profesionales en las universidades. Consideraciones y acciones hoy, 2019-2024. Ecuador

Aguilera, García, L, O (2018) Sistematización de experiencias de desarrollo local, Holguín, Cuba: Editorial INKJET

Asamblea Nacional de la República del Ecuador, Ley Orgánica de Educación Superior,(2010), https://goo.gl/S2L9BF, (en línea), 20 de diciembre de 2016, Asamblea Nacional, Registro Oficial Año II, № 298,12 de octubre de 2010, Quito, Ecuador

Área de convenio de Relaciones internacionales y Redes académicas, Convenio marco de cooperación interinstitucional entre la Universidad Estatal del Sur de Manabí y la Universidad Técnica de Manabí (2015).26 de agosto

Davyt , A y Cabrera, C,(2014) Vinculación Universidad - Sociedad y formaciones universitarias: una perspectiva histórica y una tesis actual , Congreso Iberoamericano de Ciencia, Tecnología, Innovación y Educación, artículo 716, Buenos Aires, Argentina

(C) Universidad Estatal del Sur de Manabí. Jipijapa, Ecuador. 
Resabala Claribel Silvia González Calzadilla; Manuel de Jesús Andèrez Velázquez; Karen Lorena Briones Cedeño

Elisa Bernal CEPAL

https://www.cepal.org/ilpes/noticias/paginas/1/36861/Presentacion_Experiencias_innovacion_social.pdfaría

Hernández-Ascanio, j., Tirado Valencia, p. \& Ariza-Montes, a. (2016): “El concepto de innovación social: ámbitos, definiciones y alcances teóricos”, CIRIEC-España, Revista de Economía Pública, Social y Cooperativa, 88, 165-199.

Herrera Gutiérrez María Rosa Innovación social comunitaria: miradas a una experiencia de ocupación de vivienda1

Malagón Plata, L. A. (2006) La vinculación Universidad-Sociedad desde una perspectiva social, https://goo.gl/L31pAQ, Rev. Educación y Educadores ISSN 0123-1294, Volumen 9, Número 2, pp. 79-93

Moulaert, F. (2013). Social innovation: intuition, precept, concept, theory and practice. En: F. Moulaert et al. (eds.), The International Handbook on Social Innovation. Collective Action, Social Learning and Transdisciplinary Research (pp. 13-24). Cheltenham, Edward Elg

Margaschmidt https://transperiencias.com/2017/06/14/el-proceso-de-escucha-en-la-innovacion-social/

Núñez Jover, J, Alcanzar Quiñones, A, (2016) ¿Universidad y desarrollo Local/territorial? Argumentos conceptuales y sugerencias para las políticas institucionales, La Habana: Editorial Félix Varela,

Rozas Pazaga, M. (2001). La intervención profesional en relación con la cuestión social. El caso del Trabajo Social. Buenos Aires: Espacio Editorial

UNESCO (1995). Conferencia Regional sobre Políticas y Estrategias para la transformación de la educación Superior en América latina y El Caribe. Caracas: UNESCO.

Figaredo, F. (2009). La emergencia ciencia, tecnología, sociedad. La Habana: Universidad La Habana. 